\title{
Venom phospholipases of Russell's vipers from Myanmar and eastern India-Cloning, characterization and phylogeographic analysis
}

\author{
Inn-Ho Tsai ${ }^{\mathrm{a}, *}$, Hsin-Yu Tsai ${ }^{\mathrm{a}}$, Ying-Ming Wang ${ }^{\mathrm{a}}$, Tun-Pe $^{\mathrm{b}}$, David A. Warrell ${ }^{\mathrm{c}}$ \\ a Institute of Biological Chemistry, Academia Sinica, and College of Life Sciences, National Taiwan University, Taipei 106, Taiwan \\ ${ }^{\mathrm{b}}$ Department of Medical Research, Yangon, Myanmar \\ ${ }^{c}$ Nuffield Department of Clinical Medicine, John Radcliffe Hospital, Headington, Oxford OX3 9DU, UK
}

Received 19 February 2007; received in revised form 22 April 2007; accepted 24 April 2007

Available online 5 May 2007

\begin{abstract}
Venoms of Russell's vipers (genus Daboia) are known for their deadly coagulopathic and other effects. We herein studied various isoforms of venom phospholipases $\mathrm{A}_{2}$ (PLAs) from two Daboia species at their geographic boundary. From Myanmar Daboia siamensis venom (designated as DsM), four PLAs (designated DsM-aI, aI', aII' and bI') were purified, and the cDNAs encoding two acidic (DsM-aI and aII) and two basic PLAs (DsM-bI and S1) were also cloned from its venom-glands. DsM-S1 is identical to the major venom PLA of southern India Daboia russelii, but the protein is absent from the venom. Additionally, four PLAs (designated DrK-aI, aII, bI and bII) were cloned from cDNA obtained from venom glands of a Kolkata D. russelii, and the PLAs were purified from the pooled venom (designated as DrK). The acidic DrK-aI is the most neurotoxic and lethal among these PLAs; DsM-aI which differs from DrK-aI by only the Phe2 substitution shows greatly reduced enzymatic activity and lethality. Both acidic PLAs do not form dimeric complex with basic PLAs in the same venoms. DsM-bI' is neurotoxic and lethal but its orthologous DrK-bI (97\% identical to DsM-bI') is a much weaker toxin. Given the fact that most of the orthologous PLAs of DrK and DsM share $97-100 \%$ sequence identity, Daboia vipers of Myanmar and Kolkata must be closely related. Molecular phylogenetic analyses on 30 venom PLAs of Eurasian vipers' revealed co-evolution of five subtypes of venom PLAs in both Daboia and Vipera genera. Our results shed light on the intra- and inter-species variations and structure-function relationships of viperid venom PLAs.
\end{abstract}

(C) 2007 Elsevier B.V. All rights reserved.

Keywords: Venom phospholipase $\mathrm{A}_{2}$; Cloning and sequencing; Phylogenetic analysis; Geographic variation; Russell's viper (Daboia siamensis, Daboia russelii)

\section{Introduction}

Russell's vipers (Viperinae of the genus Daboia) are important causes of morbidity and mortality in South and Southeast Asia. Two species of Daboia, Daboia russelii [1] and Daboia siamensis [2], have been identified. D. russelii is mainly distributed in the Indian sub-continent while $D$.

Abbreviations: DsM, Daboia siamensis (Myanmar); DrK, Daboia russelii (Kolkata); dPPC, L-dipalmitoyl phosphatidylcholine; PLA, Phospholipase $\mathrm{A}_{2}$; HPLC, high performance liquid chromatography

is GenBank accession numbers for the novel nucleotide sequences of venom PLAs are DQ090654-7 for those from D. siamensis (Myanmar), and DQ090658-61 for those from D. russelii (Kolkata).

* Corresponding author. POB 23-106, Institute of Biological Chemistry, Academia Sinica, Taipei, Taiwan. Tel.: +886 22 3620264; fax: +886 22 3635038

E-mail address: bc201@gate.sinca.edu.tw (I.-H. Tsai). siamensis is discontinuously spread over a wide area from Myanmar to Taiwan and south to Indonesia. Clinical effects and antigenicities of Daboia venoms vary throughout these different geographic ranges [2-8]. Envenoming by D. siamensis poses a remarkably severe problem especially in Myanmar where it has been the country's 5th leading single cause of death [3-5]. Clinical observations also indicated higher case fatalities and more severe hemorrhagic manifestations in Daboia envenoming in Myanmar and eastern India than in other regions [2,6-9].

Snake venom is the richest source of secreted phospholipases $\mathrm{A}_{2}$ (PLA; EC 3.1.1.4), the $\mathrm{Ca}^{+2}$-dependent enzymes that hydrolyzes the 2-acyl ester of phosphoacylglyceride $[9,10]$. Paralogous PLA variants in viperid venoms have been derived from gene duplication and rapidly evolved to acquire functional diversity [11-13]. Up to $65 \%$ of the venom proteins of Daboia from Taiwan, Thailand, southern India and Pakistan 
are PLAs, many of them have been cloned or fully sequenced [14-16]. Notably, most of the lethal or neurotoxic PLAs found in snake venom are basic proteins, but two acidic PLAs of high lethal potency have recently been purified from Daboia venoms of eastern India [9] and Myanmar [17,18]. Since their complete sequences were not solved, the structureactivity relationships of both PLA-toxins were difficult to understand.

The aim of the present study is to resolve structures and functions of novel venom PLAs from Daboia subspecies and better understand their geographic variations. We thus purified, characterized and cloned venom PLA isoforms of Daboia vipers from Myanmar (i.e. Burma) and Kolkata (eastern India), at the boundary between $D$. russelii and $D$. siamensis ranges. The results were compared with the venom PLA data of Daboia from adjacent areas, including Pakistan, southern India, Sri Lanka, Thailand, and Taiwan [16-19]. By phylogenetic analyses and sequence alignments of 30 Viperinae venom PLAs, the classification and structure-function relationships of these PLAs are discussed.

\section{Materials and methods}

\subsection{Venoms and other materials}

D. siamensis venom samples from southern Myanmar were kindly given by Prof. R.D.G. Theakston, Liverpool School of Tropical Medicine (UK) while another pooled sample from northern Myanmar was obtained from Prof. Y. Y. Shu of Kuangxi Medical University, China. Meanwhile, live specimens of Daboia were also caught near Yangon (Myanmar) and Kolkata (eastern India), respectively. Fresh venom glands were dissected immediately from each snake after they were euthanized $48-60 \mathrm{~h}$ after venom collection. The glands were preserved for several weeks in the RNAlater solution (Ambion, USA) prior to RNA extraction.

Modifying enzymes, restriction enzymes and the pGEM-T vector were purchased from Promega Corp. (Madison, USA). Synthetic L-dipalmitoyl phosphatidylcholine (dPPC) was purchased from Avanti Polar Lipids (Alabaster, AL, USA). Triton X-100, sodium deoxycholate, acetylcholine, and other chemicals were of reagent grade from either Merck (Darmstadt, Germany) or Sigma (St. Louis, USA).

\subsection{Purification of venom PLA}

Crude Daboia venom was dissolved in $0.1 \mathrm{M}$ ammonium acetate buffer $(\mathrm{pH}$ 6.5) and then fractionated on a FPCL equipped with a Superdex G75 column (HR10/30, Pharmacia) pre-equilibrated with the same buffer, at room temperature. The $\mathrm{PLA}_{2}$-containing factions were pooled and freeze-dried. The venom proteins were further purified by reversed-phase HPLC using a $\mathrm{C}_{18}$ column $(4.5 \times 250 \mathrm{~mm}, 10 \mu$, Vydac $)$ equilibrated with $0.07 \%$ aqueous trifluoroacetic acid (TFA, solvent A), and eluted with a linear gradient of 20 $45 \% \mathrm{CH}_{3} \mathrm{CN}$ containing $0.07 \%$ trifluoroacetic acid (solvent B). Each peak was dried in a vacuum-centrifuge device (Labconco, USA). Concentrations of crude venom and PLA in solutions were determined spectrophotometrically at $280 \mathrm{~nm}$, assuming an extinction coefficient of 1.5 at $1.0 \mathrm{mg} / \mathrm{ml} \mathrm{[14]} \mathrm{and} \mathrm{method} \mathrm{of}$ Bradford [20].

\subsection{Protein sequences and masses}

Purity of venom protein was assessed by SDS-PAGE and $N$-terminal sequencing. The $N$-terminal sequence of each purified protein was determined by an Applied Biosystems amino acid sequencer (Model Procise 492) [21]. Its molecular weight was analyzed by QSTAR XL nano-ESI mass spectrometer System (Applied Biosystems, Foster City, USA).

\subsection{Cloning and sequencing}

One Daboia specimen from each region was euthanized 2 days after venom extraction, at which point the venom glands were removed immediately for RNA extraction. The mRNA was prepared and the cDNA synthesis kits (Stratagene, USA) was used, as previously described [14]. Using venom gland cDNA as a template, the PCR [22] was conducted using SuperTaq DNA Polymerase with a pair of pairs of mixed-base oligonucleotide primers (sense primer 1: TCTGGATTSAGGAGGATGA GG; antisense primer 2: GCCTGCAGRACTTAGCA), that were specifically designed based on conserved $3^{\prime}$ and $5^{\prime}$ untranslated regions of several homologous cDNA encoding other viperid venom PLAs [12,14]. In addition, a sense primer 3 (GCGGAGATGATCGTNAARATG) based on the amino acid sequence AEMIVK was used in conjunction with primer 2 in an attempt to specifically amplify Drk-aI' and DsM-aI'.

After treatment with polynucleotide kinase, the amplified DNA fragment was inserted into the pGEM-T easy vector (Promega Biotech, Wisconsin, USA). It was then transformed into Escherichia coli strain JM 109. Only the white transformants were picked-up and the cDNA clones were selected by restriction enzyme gel pattern. The DNA sequencing System model 373A and the TaqDye-Deoxy terminator cycle sequencing kit (PE Applied Biosystems, USA) were used to determine the cDNA sequences by dideoxynucleotide method [23]. Full amino acid sequences of venom PLAs were deduced from the nucleotide sequences of cDNA.

\subsection{Enzymatic activities and pharmacological effects}

$\mathrm{PLA}_{2}$ activities were measured by $\mathrm{pH}$-stat titration method at $37^{\circ} \mathrm{C}$ on a $\mathrm{pH}-$ stat apparatus (Radiometer RTS 822, Copenhagen, Denmark). Released fatty acids, from $3 \mathrm{mM}$ L-dipalmitoyl phosphatidylcholine (dPPC) mixed either with an equal concentration of deoxycholate or two fold concentration of Triton X100 in $0.10 \mathrm{M} \mathrm{NaCl}$, were titrated at $\mathrm{pH} 7.4$ with $6 \mathrm{mM} \mathrm{NaOH}$. The initial rate was recorded for more than five $\min$ and corrected for non-enzymatic spontaneous rate. Specific activity was expressed as $\mu \mathrm{mol}$ of dPPC hydrolyzed per min per mg of the enzyme. In addition, $1.0 \%(\mathrm{w} / \mathrm{w})$ fresh egg yolk in $0.15 \mathrm{M}$ $\mathrm{NaCl}$ was used as substrate for comparing PLA activities by $\mathrm{pH}$-stat titration methods.

Induction of edema by venom PLA on hind paw of anaesthetized Wistar rats (150-160 g body mass) was monitored up to $5 \mathrm{~h}$ by a plethysmometer, as described previously [12]. To test the lethal effects of crude venom and purified PLAs, ICR mice of $30-35 \mathrm{~g}$ body weight were used $[9,24]$. The venom proteins were dissolved in sterile PBS and centrifuged at $10,000 \times \mathrm{g}$ to remove insoluble materials before injected to the mice. The medium lethal dose $\left(\mathrm{LD}_{50}\right)$ was estimated by intraperitoneal (i.p.) injection of graded doses of the protein to the mice. Effects of venom proteins on four mice in each group were examined; the lethality was estimated as the dosage-range which was able to kill about $50 \%$ of the test animals within $24 \mathrm{~h}$.

\subsection{Phylogenetic analyses}

Amino acid sequences of venom PLAs from various species under Viperinae subfamily were retrieved by Blast search [25] and aligned along with those solved in the present study by AlignX in VectorNTI (Invitrogen, USA) program. Cladograms were constructed based on these sequences by neighbor-joining algorithm using program PHYLIP [26]; degree of confidence for the internal linage of the tree was determined by bootstrap methods [27].

\section{Results}

\subsection{Purification and biochemical characterization}

Patterns of gel filtration of the crude venoms by Superdex G75 column on a FPLC system were shown in Fig. 1. The PLAcontaining fractions were lyophilized and further purified by reversed-phase HPLC (not shown). Four venom PLAs could be 


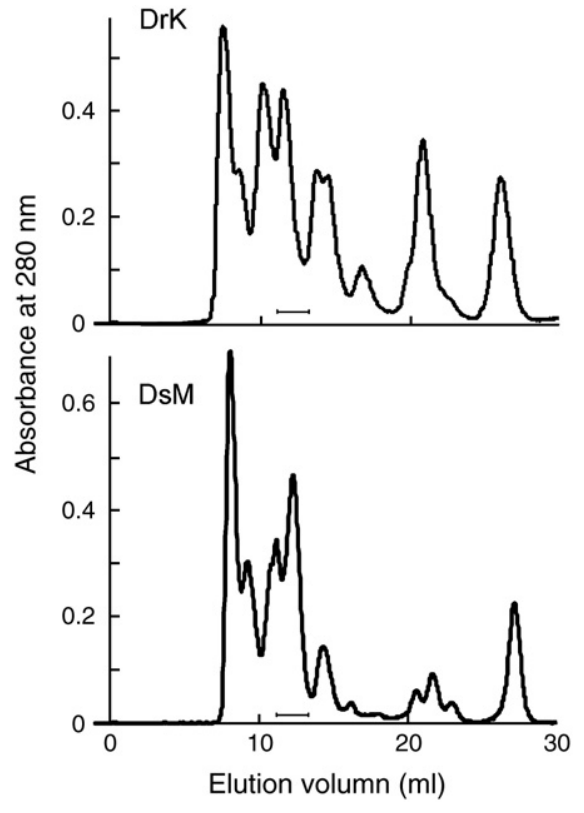

Fig. 1. Gel filtration of the crude venom of DrK and DsM. Crude venom was dissolved and injected into a Superdex G75 (HR10/30) column on a FPLC system. The elution was carried out with the equilibration buffer, 0.1 M ammonium acetate $(\mathrm{pH} \mathrm{6.4})$, at a flow rate of $1.0 \mathrm{ml} / \mathrm{min}$. Fractions of $1.0 \mathrm{ml}$ were collected. The PLA-containing fractions (shown by bars) were pooled.

purified and identified from each of DrK and DsM (northern Myanmar). Their protein masses, N-terminal sequences and enzyme activities were also determined, as listed in Table 1. The PLA isoforms were designated according to their $\mathrm{pI}$ values deduced from the protein sequence ( $a$, for acidic; $b$ for basic), as well as the N-terminal sequence homology. In addition, small amount of the crude venom was fractionated by HPLC directly (Fig. 2) to confirm the PLA-profiles obtained by the two-step procedure.

The acidic DrK-aI is the most neurotoxic and lethal among these PLAs, similar to a previous report [9]. Notably, DrK-aI showed strong preference for the anionic substrate over the zwitterionic substrate (Table 1). This is consistent with previous findings for other venom presynaptic neurotoxins, including $\beta$ bungarotoxin, crotoxin [28] and ammodytoxin [29]. Notably, several PLA isoforms of DsM and DrK bear a Phe2 substitution which was seldom observed in other venom PLAs, and they all showed much lower catalytic activities than the PLAs containing Leu2 when both types of micellar substrates were used in vitro (Table 1). When $1.0 \%(\mathrm{w} / \mathrm{w})$ solution of egg yolk was used as substrate, DrK-aI had a low specific activity of $23 \mu \mathrm{mol} / \mathrm{min} /$ $\mathrm{mg}$ at $37^{\circ} \mathrm{C}$, while the activities of the Phe2-PLAs (DsM-aI and Drk-aI') were too low to be measured.

Since cDNA had been obtained from venom glands of a $D$. siamensis specimen collected near Yangon (southern Myanmar), PLAs were also purified from several venom batches of D. siamensis specimens, which had been collected in the same area (supplied by Prof. R.D.G. Theakston). HPLC profiles, masses, and N-terminal sequences of the PLA isoforms in these samples suggested that the PLAs are present in different proportions and possibly show some individual variations. Each of the venom sample contains four or five PLA-variants, mass of the major PLAs have been determined to be 13607, 13810, 13830, and 14084 Da, respectively. Thus, DsM-PLA variant with mass of 13830 appears to be present in Daboia venom of southern Myanmar and DrK but not that of northern Myanmar (Table 2).

\section{2. cDNA cloning and protein sequence alignments}

PCR has been carried out using specifically designed primers for venom PLAs with the venom gland cDNAs as templates $[12,14]$. After the cDNAs were amplified and cloned, their nucleotide sequences were determined to predict the PLA protein sequences and $\mathrm{pI}$ values. Based on sequencing more than 45 selected PLA clones for each species, sequences of four distinct Asp49 PLAs, respectively, could be deduced from the cDNA sequences of both DrK and DsM (Table 2). Their complete amino acid sequences were then aligned with those of most related venom PLAs obtained by BlastP search [25]. Three putative categories of the Asp49-PLAs from Viperinae venoms (i.e. acidic Asn1, basic Asn1, and Ser1) were aligned in Fig. 3A, $\mathrm{B}$ and $\mathrm{C}$, respectively.

Most of the cDNA deduced sequences (Table 2) could match those of the purified PLA isoforms (Table 1), except that the masses of purified DsM-aII' (13810) and DsM-bI' (14084) do not match DsM-aII (13830) and DsM-bI (14055) predicted from the cDNA sequences, respectively, though their N-terminal sequences compromised. Further PCR experiments using primers 3 and 2, followed by cloning and sequencing of another 20 cDNAs, failed to produce any new clones encoding DsM-aI'. It lays the possibility that the two PLA pairs, DsM-aII and aII', DsM-bI and bI', are allelic proteins from different individual snakes or geographic samples.

\subsection{Lethal and edematous effects}

DrK and DsM appear to evolved functionally different PLAs, in spite of the fact that their PLA sequences are very

Table 1

Characterization of purified venom PLAs from DrK and DsM venoms

\begin{tabular}{|c|c|c|c|c|c|}
\hline \multirow[t]{2}{*}{$\begin{array}{l}\text { Venom } \\
\text { PLA }\end{array}$} & \multirow{2}{*}{$\begin{array}{l}\% \\
\text { content } \\
(\mathrm{w} / \mathrm{w})\end{array}$} & \multirow[t]{2}{*}{$\begin{array}{l}\text { Mass, } \\
\text { Da }\end{array}$} & \multicolumn{2}{|c|}{$\begin{array}{l}\text { Specific activities }{ }^{\mathrm{a}} \\
(\mu \mathrm{mol} / \mathrm{mg} / \mathrm{min})\end{array}$} & \multirow[t]{2}{*}{$\begin{array}{l}\text { N-terminal sequence } \\
\text { determined }\end{array}$} \\
\hline & & & deoxycholate & $\begin{array}{l}\text { Triton } \\
\mathrm{X}-100\end{array}$ & \\
\hline DrK-aI & 17 & 13573 & $1127 \pm 58$ & $196 \pm 15$ & NLFQFAEMIVK \\
\hline DrK-aI' & 7 & 13812 & $24 \pm 1$ & 5.8 & NFFQFAEMIVK \\
\hline DrK-bI & 6 & 14077 & $2352 \pm 224$ & $614 \pm 36$ & NLFQFARMINQ \\
\hline DrK-bII & 0.2 & 13704 & $1150 \pm 22$ & $42 \pm 0.9$ & NLLQFGRMINQ \\
\hline DsM-aI & 12 & 13607 & $\underline{10}$ & 6.2 & NFFQFAEMIVK \\
\hline DsM-aI' & 4 & 13812 & $\overline{29} \pm 2$ & 7 & NFFQFAEMIVK \\
\hline DsM-aII' & 7 & 13810 & $1538 \pm 25$ & $320 \pm 4$ & NLYQFGEMINQKT \\
\hline DsM-bI' & 12 & 14084 & $1131 \pm 26$ & $622 \pm 26$ & NLFQFARLIDA \\
\hline
\end{tabular}

Masses were determined by ESI-MS spectrometry.

a Enzymatic hydrolysis of $\mathrm{dPPC}$ were measured in the presence of deoxycholate or Triton X-100 with $10 \mathrm{mM} \mathrm{CaCl}_{2}$ at $37{ }^{\circ} \mathrm{C}$. Values shown are median \pm S.E. of results from three independent experiments, and underlined values are averages of two experimental results. 


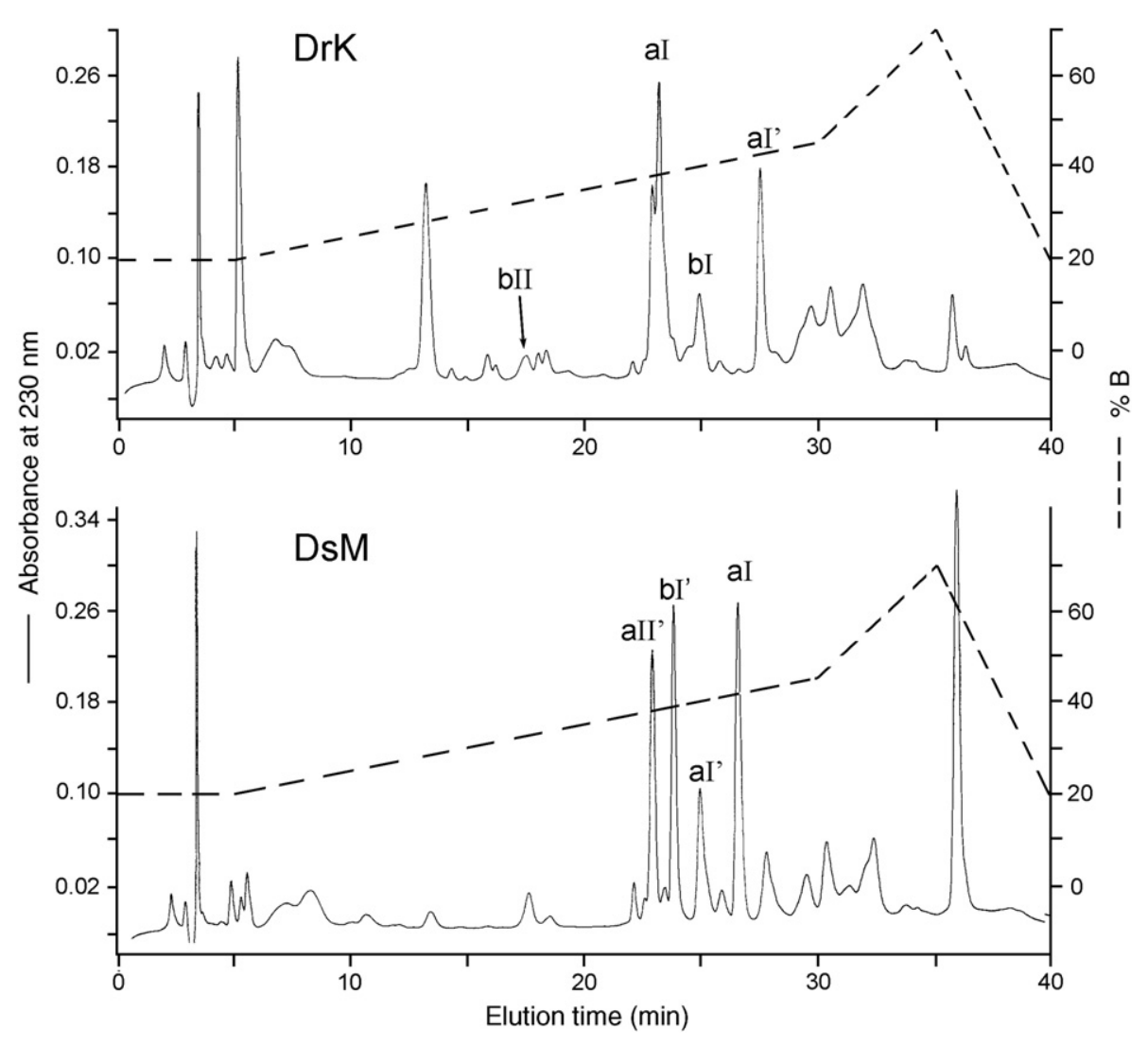

Fig. 2. Purification of PLAs by reversed-phase HPLC. Solubilized crude venoms of DrK and DsM in solvent A were fractionated on a $\mathrm{C}_{18^{-}} \mathrm{Vydac}_{\mathrm{HPLC}}$ column. The PLA-containing peak was identified by ESI-MS and N-terminal sequencing and annotated (as shown in Table 1).

similar. Purified DrK-aI at doses between 0.06 and $0.10 \mu \mathrm{g} / \mathrm{g}$ was lethal to about half of the mice in $24 \mathrm{~h}$. In contrast, DsM-aI, which differed from DrK-aI by only residue 2, had very low enzymatic activity (Table 1) and was not so toxic to mice

Table 2

cDNA data for venom PLAs of DrK and DsM

\begin{tabular}{|c|c|c|c|c|}
\hline $\begin{array}{l}\text { PLA } \\
\text { cloned }\end{array}$ & $\begin{array}{l}\text { Calculated mass } \\
(\mathrm{Da})\end{array}$ & $\mathrm{pI}$ & $\begin{array}{l}\text { No. of } \\
\text { clones }\end{array}$ & $\begin{array}{l}\text { (Signal peptide) and } \\
\text { residues } 1-10\end{array}$ \\
\hline DrK-aI & 13573 & 4.6 & 3 & $\begin{array}{l}\text { (MRTLWIVAVCLIGVEG) } \\
\text { NLFQFAEMIV }\end{array}$ \\
\hline DrK-aII ${ }^{\mathrm{a}}$ & 13830 & 4.9 & 4 & $\begin{array}{l}\text { (MRTLWIVAVCLIGVEG) } \\
\text { NLYQFGEMIN }\end{array}$ \\
\hline DrK-bI & 14076 & 8.7 & 5 & $\begin{array}{l}\text { (MRTLWIVAMCLIGVEG) } \\
\text { NLFQFARMIN }\end{array}$ \\
\hline DrK-bII & 13704 & 8.4 & 3 & $\begin{array}{l}\text { (MRTLWIVAVCLIGVEG) } \\
\text { NLLQFGRMIN }\end{array}$ \\
\hline DsM-aI & 13607 & 4.6 & 11 & $\begin{array}{l}\text { (MRTLWIMAVCLIGVEG) } \\
\text { NFFQFAEMIV }\end{array}$ \\
\hline DsM-aII ${ }^{\mathrm{a}}$ & 13830 & 4.9 & 2 & $\begin{array}{l}\text { (MRTLWIVAVCLIGVEG) } \\
\text { NLYQFGEMIN }\end{array}$ \\
\hline DsM-bI & 14055 & 8.7 & 2 & $\begin{array}{l}\text { (MRTLWIVAMCLIGVEG) } \\
\text { NLFQFARLID }\end{array}$ \\
\hline DsM-S1 ${ }^{\text {a }}$ & 13625 & 8.4 & 2 & $\begin{array}{l}\text { (MRTLWIVAVCLIGVEG) } \\
\text { SLLEFGKMIL }\end{array}$ \\
\hline
\end{tabular}

Isoelectric point $(\mathrm{pI})$ and molecular mass were predicted from the deduced protein sequence.

a cDNA deduced proteins failed to match those listed in Table 1.
$\left(\mathrm{LD}_{50}>3.0 \mu \mathrm{g} / \mathrm{g}\right)$. All the mice injected with DsM-aI survived in our experiments but were hypo-locomotive the first few hours. We also compared the edema-inducing effects of DsM-aI and DrK-aI on rat hind paws. Both PLAs at the dose of $10 \mu \mathrm{g}$ effectively induced edema but DrK-aI had a stronger and faster effect than DsM-aI. Relative swelling of paw was peaked in 4 or $5 \mathrm{~h}$ after the injection, and reached $38.5 \%$ and $30.3 \%$ for DrK-aI and DsM-aI, respectively (Fig. 4).

While toxicity of DsM-aI was weak, the basic DsM-bI' in the same venom was more neurotoxic and lethal and its toxicity was not affected or enhanced by the presence of DsM-aI (Table 3). Notably, DrK-bI has good enzyme activity but it is not as lethal as DsM-bI' to mice although both PLAs share about 97\% sequence identity (Fig. 3B).

\subsection{Phylogenetic analyses}

Cladogram was established based on the amino acid sequences of venom PLAs from selected Eurasia Viperinae including most Vipera and Daboia. A basic venom G6D49PLA from Trimeresurus puniceus (an Indonesia pitviper) was used as the out-group (Fig. 5). Remarkably, this robust tree of Viperinae venom Asp49-PLAs shows that the PLAs with Asn at the $N$-terminus (designated as N1) form a distinct cluster separated from those with Ser at the $\mathrm{N}$-terminus (designated as S1). In the N1-PLA cluster, at least four subtypes of PLAs with distinct $N$-terminal sequences are selectively present in various 
Vipera and Daboia venom species (Fig. 5), including two acidic subtypes with either $\mathrm{F} 3$ or $\mathrm{Y} 3$ (or $\mathrm{T} / \mathrm{S}$ 3) substitution, and a highly basic subtype containing A11K12 and a less basic subtype containing K11M12.

\section{Discussion}

Contents of venom PLAs in DsM and DrK (28-38\% of the venom proteins by weight) are lower than those in Daboia

A

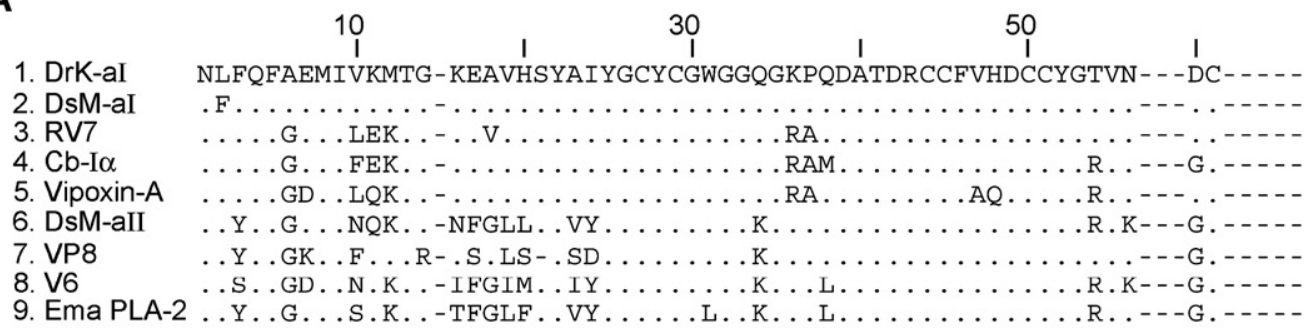

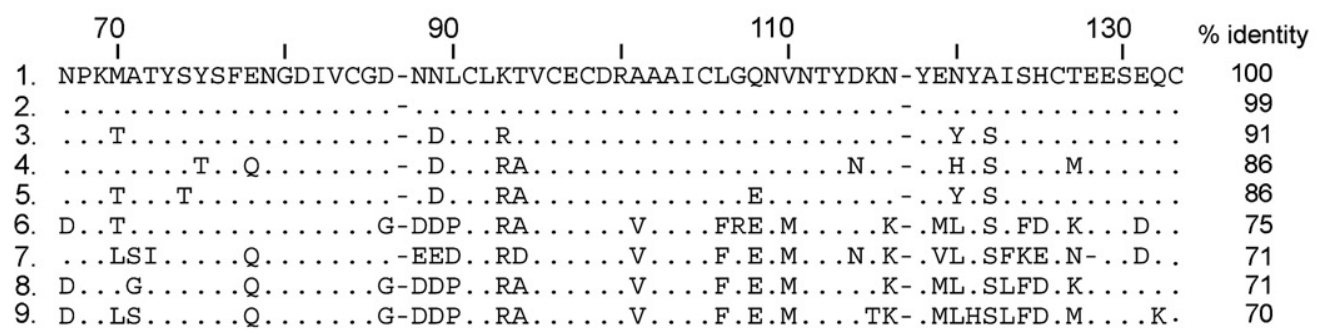

B

\begin{tabular}{lll} 
& \multicolumn{1}{l}{10} &
\end{tabular}

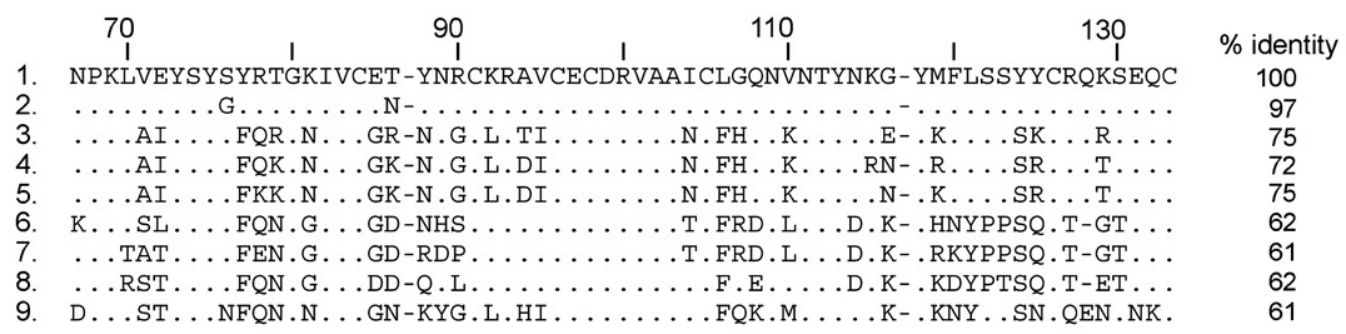

C

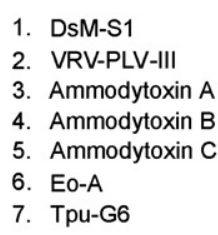

Tpu-G6

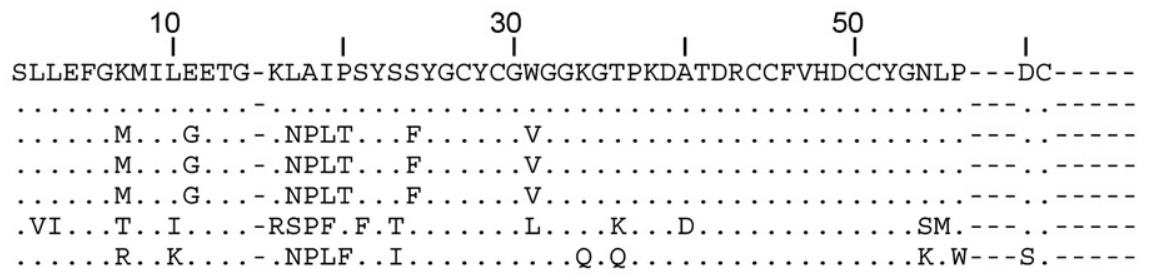

n
130

\section{0}

$\%$ identity 100

99 81 


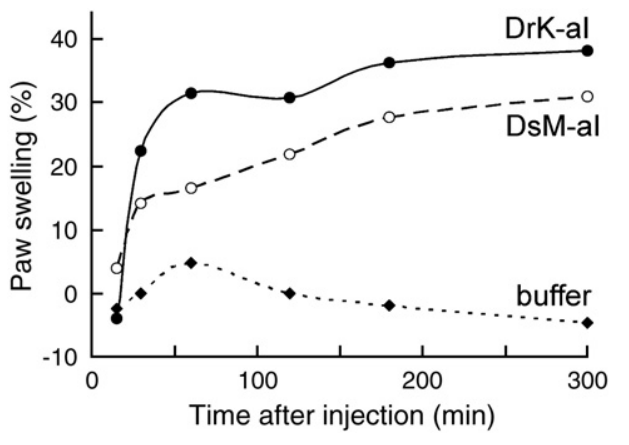

Fig. 4. Oedema-inducing activities of DrK-aI and DsM-aI on rat paw. Time course of the swelling of rat hind-paw was followed after injection of $10 \mu \mathrm{g}$ of the PLA dissolved in $100 \mu$ l PBS. Volume of the paw was measured by a plethysmometer, \% paw swelling (relative to the volume measured 1 min after the injection) was the average from results of two independent experiments.

venoms from Thailand, China, Taiwan and Pakistan (55-65\% of the total proteins). Various effects elicited by the Daboia PLAs have been reported, including neurotoxic [14,17], cytotoxic, myotoxic [16], hypotensive, and/or anticoagulating [30], and some PLAs may show more than one of the effects. In the present study, four PLAs were purified from each of DrK and DsM, and eight novel venom PLAs have been cloned and fully sequenced (Table 2, Fig. 3). PLAs of all geographic venom samples of Daboia are found to contain Asp49, and many of the DrK and DsM PLAs contain rather unique Ala6 substitution.

We previously reported the $\mathrm{N}$-terminal sequences of five PLAs purified from D. siamensis (formerly Vipera r. siamensis) venom purchased from Miami Serpentarium (Florida, USA) [19]. However, our later analyses of Daboia venom obtained from Thai Red-Cross Society, Bangkok revealed that it contained only the RV4/RV7 heterodimeric PLA (unpublished results), just like Taiwan D. siamensis venom [14]. This was supported by another cloning study using Thai $D$. siamensis venom glands [15]. In fact, the $N$-terminal sequences of three minor PLAs (S1-2, S1-1 and S3) in previous report [19] are identical to those of DsM-aI, DsM-aII and DsM-bI, respectively (Table 1 ). Thus, the $D$. siamensis venom from Miami Serpentarium was heterogeneous and possibly contained $20 \%$ of the venom from Myanmar.

Results in Tables 1 and 2 are helpful for the identification and matching of purified PLAs with their cDNA clones. However, masses of purified DsM-aI' and of DsM-bI' cannot exactly match those of DsM-aI and of DsM-bI (deduced from the cDNA data). The discrepancies could be attributed to the fact that we used venom glands of Daboia specimen from southern Myanmar for cDNA cloning, but the pooled northern Myanmar Daboia venom was used for protein analyses. We suspect that the PLA pairs, DsM-aI and aI', DsM-bI and bI', are probably
Table 3

Lethal potencies of crude venom and purified PLAs on ICR mice

\begin{tabular}{ll}
\hline Venom or PLA & Lethality, (i.p.) $\mu \mathrm{g} / \mathrm{g}$ mouse body weight \\
\hline DrK-aI & $0.06<\mathrm{LD}_{50}<0.13$ \\
DsM-aI & $\mathrm{LD}_{50}>3.0$ \\
DrK-bI & $\mathrm{LD}_{50}>3.0$ \\
DsM-bI' & $0.13<\mathrm{LD}_{50}<0.20$ \\
DsM-aI+DsM-bI' $(1: 1)$ & $0.40<\mathrm{LD}_{50}<0.50$ \\
DrK soluble venom & $1.0<\mathrm{LD}_{50}<1.33$ \\
DsM soluble venom & $0.3<\mathrm{LD}_{50}<0.4$ \\
\hline
\end{tabular}

allelic proteins from different geographic samples. However, possibilities that we did not obtain all the transcripts of PLA from DsM venom glands, or that some of purified PLAs have undergone post-translational modification or processing, could not be ruled out. In addition, Blast-search helped to detect similar data recently reported by other researchers after our data have been deposited. For examples, D. r. siamensis PLA-III (AY303800) and PLA-II (AY286006) appear to be identical to DsM-aI and DsM-bI, while D. r. russelii PLA-I (DQ365974) and PLA-II (DQ365975) are identical to DrK-aI and DrK-bI, respectively. Another clone (AY256974) in the databank was possibly a hybrid of DsM-aII and aI, and the other (DQ365977) appeared to be a hybrid of DrK-aI and bII, they possibly derived from artifacts of PCR experiments.

Envenoming by both DsM and DrK was reported to be more hemorrhagic than by Daboia specimens from other geographic areas $[3-5,7,8]$, which is attributed to special expression of haemorrhagic metalloproteinases in both DsM and DrK (results to be published). Except for the absence of a DrK-bII homolog in DsM (Table 1), all the deduced DsM-PLA sequences are $\geq 97 \%$ similar or identical to their orthologous PLAs in DrK (Fig. 3), and a PLA-isoform identical to DsM-aI is also present in low content in DrK. These venom similarities suggest that Daboia vipers from Myanmar and eastern India are closely related species and constitute a special lineage of the Daboia populations. Results of previous studies on western India $D a$ boia venom (supplied by Haffkine Institute, Mumbai) also showed that an acidic PLA (FrIII-3, pI 4.2) had most potent neuromuscular blocking action among the five PLAs purified [31]. However, venom differences between the eastern and western India D. russelii have been reported $[7,8]$.

Morphological differences between the $D$. siamensis populations of Thailand and Myanmar [32] have been reported, and heterologous antivenoms in four times greater dosages were found to be necessary to neutralize toxic effects of each venom [33]. The fact that DsM is more similar to DrK than to the D. siamensis venoms from Thailand, China and Taiwan might be explained by the notion that dispersal of Daboia "out of India" probably was

Fig. 3. Alignments of the amino acid sequences of Asp49 PLAs from Viperinae venom: (A) acidic N1-PLAs; (B) basic N1-PLAs; (C) S1-PLAs. Representative sequences were retrieved from BlastP search [25]. Single-letter codes of amino acids and the numbering system of Renetseder et al. [51] are used. Residues identical to those in the top line are denoted with dots, gaps are marked with hyphens. PLAs and the species (as the original taxonomic designation submitted) and their GenBank or SwissProt accession numbers are: C. cerastes Ccer, P21789; Vipera russelii (southern India) VRV-PL VIII, P59071; Vipera r. russelii (Pakistan) Vrr-R1, P81458; Vipera russelii formosensis RV-4 and RV-7, Q02471 and P31100; E. mafocmahonii Ema-PLA 2, P24294; Echis ocellatus Eo-A, P59171; Pseudocerastes persicus CbI, AAB36097, Cb-II, AAB36096; T. puniceus Tpu-G6D49, AAR14167; Vipera a. ammodytes Ammodytin I2, P34180, Ammodytoxin-A, B and C, P00626, P14424 and P11407; Vipera a. meridionalis Vipoxin-A, P04084, Vipoxin-B, P14420; Vipera aspis zinnikeri Vaspin-An, AF548351; Vipera b. berus V6, AY159811; Vipera palaestinae VP8 AAC78084. 


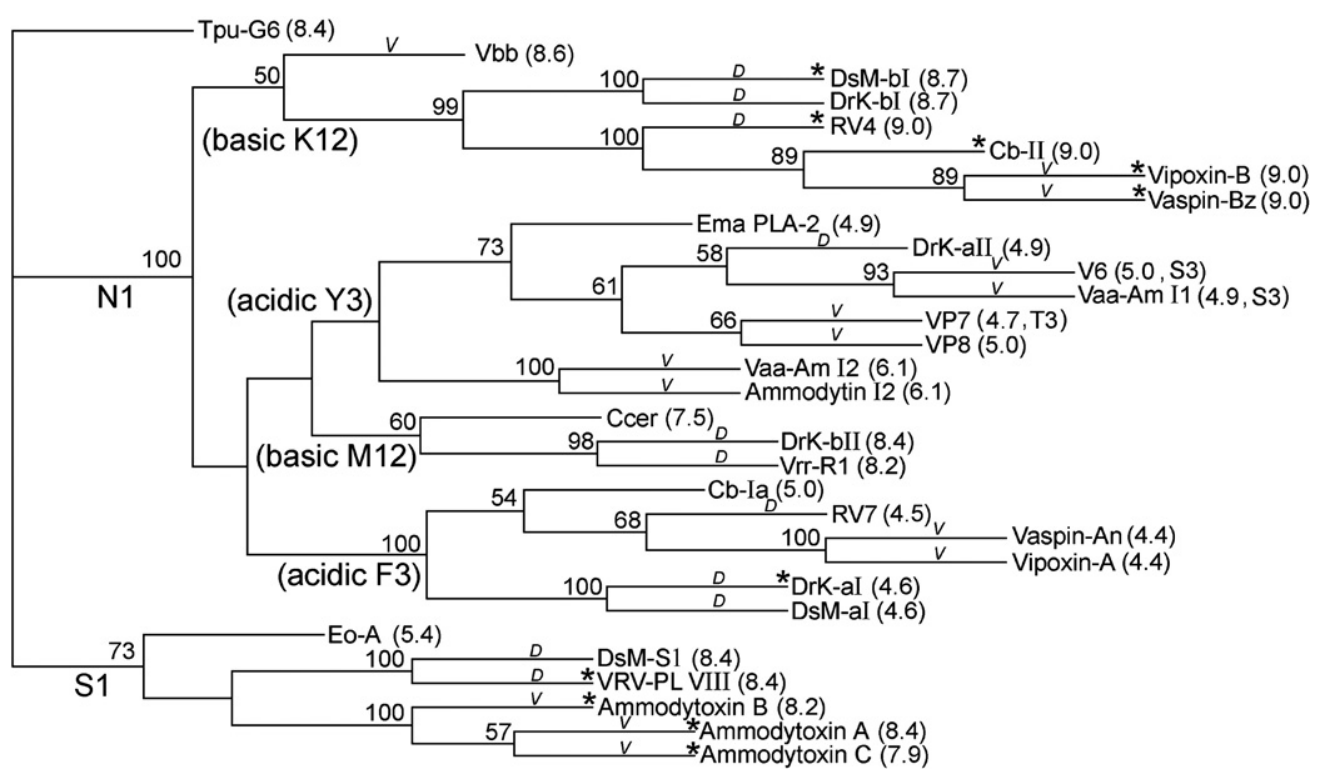

Fig. 5. Phylogenetic tree based on amino acid sequences of Viperinae venom Asp49 PLAs. The dataset includes full sequences of 30 PLAs from Viperinae venom, and a pitviper PLA (Tpu-G6D49) was used as out-group. Their pI and special N-terminal substitutions were shown in parentheses. Neurotoxic PLAs or PLAsubunits are marked with asterisks. Species names and accession numbers are: Pseudocerastes persicus Cb-II, AAB36096; Vipera a. aspis Ammodytins, Vaa-Am I1, AY159807 and Vaa-Am I2, AY158637; Vipera aspis zinnikeri Vaspin-Bz, AY158635, Vipera b. berus Vbb, P31854; Vipera palaestinae VP7, AAC78085. Values at the nodes indicate the percentage of 1000 bootstrap replicates. " $D$ or $V$ " above branches denote origin of the PLA from Daboia or Vipera venom, respectively.

hampered by the high mountain ranging from the eastern border of Myanmar. This bio-geographical distribution has also been proposed for frog and some other vertebrate groups [34].

Based on the mitochondria DNA analyses $[35,36]$, the genus Daboia is closely related to European and western Asian Vipera and Macrovipera. Moreover, both Daboia and Vipera are specifically equipped with venom procoagulating components, i.e. Factor-X and Factor-V activators [37,38]. It is interesting that their venom powder can be either white or yellow color in appearance [39], and the white venoms were less necrotic or hemorrhagic than the yellow ones [6]. Five subtypes of venom Asp49 PLA genes have been found for geographic samples of Vipera a aspis $[40,41]$ and possibly other Vipera [42]. The phylogenetic tree of PLAs in Fig. 5 unravels not only the close relationship between the two genera Daboia and Vipera but also parallel evolution of their venom PLA paralogs. It has been suggested that gene duplications followed by accelerated evolution of surface functional domains have resulted in venom PLA diversity $[13,43]$. Venom components may be differentially expressed according to geological separation [44], adaptation to prey-ecology [45] or other factors [2].

A lethal acidic PLA designated as PFIIc' was previously purified from eastern Indian Daboia venom [9]. DrK-aI probably is PFIIc' because they share identical $N$-terminal sequences and high content in the venom (15\% by weight), and are highly lethal to mice. In contrast, the most lethal PLA of DsM is the basic DsM-bI', which is present in equal abundance as DsM-aI (Table 3). Notably, enzymatic activity and toxicity of DsM-bI' were not affected by the addition of equal concentration of DsM-aI (Table 3), thus DsM-aI does not play a chaperon role. The venom content of DrK-bI is much lower than that of DrK-aI
(Fig. 2), they possibly do not associate into dimers either. It is found that DrK-bI is much less lethal than DsM-bI or bI' (Table 3) although they differed by only four residues (Fig. 3B). Since Thr41 has been conserved in all the neurotoxic or myotoxic PLAs listed in Fig. 3 as well as crotoxin B and agistrodontoxin [11] while Ser41 is usually present in less toxic PLAs and crotoxin A, it is speculated that the substitution of Thr41 might be one of the reasons behind the lower toxicity of DrK-bI relative to DsM-bI or bI'.

In both pancreatic and snake venom PLAs, Leu2 is highly conserved [46], and probably is important for binding of the substrates fatty acyl chain. Mutagenesis of Leu2 to Trp2 in pancreatic PLA resulted in 33-folds decrease of the enzymatic rate [47]. DsM-aI differs from DrK-aI only at residue 2, the $40-45$ folds reduction in the catalytic rates and the lethality of DsM-aI relative to DrK-aI thus can be solely attributed to its Phe2-substitution (Table 1). The hydrolytic products of neurotoxic PLAs are suggested to be crucial for their presynaptic toxicities [48]. Mutants of PLA-neurotoxins with lower catalytic activities than the native form usually showed decreased toxicities $[29,49]$. The $N$-terminal structures of PLA have also been known to be critical for the neurotoxicities $[49,50]$. Thus, single amino acid substitution at strategic position of a venom PLA toxin may greatly affect its biological functions.

Having an identical $N$-terminal sequence to that of daboiatoxin previously purified from Myanmar D. siamensis venom [17,18], DsM-aI is much less lethal than daboiatoxin (Table 3). This discrepancy and the reason why the viper expresses such inactivated DsM-aI remain puzzling. Although DsM-aI showed significant edematous effect (Fig. 4), its other functions remain to be investigated. Moreover, the sequence of 
DrK-aI is $91 \%$ identical to that of RV7 from Taiwanese Daboia siamensis venom (Fig. 3A). The substitutions of about ten amino acid residues in RV7, including E7K, T70M and D89N, Y120N, could transform this non-lethal chaperone-like subunit [14] to a highly lethal and neurotoxic DrK-aI, or vise versa. These naturally occurring isoforms with minor structural differences but great functional variations thus provide a new platform for further investigation of structure-function relationships of venom PLAs.

\section{Acknowledgments}

We are grateful to Prof. Antony Gomes (Univ. of Calcutta) for his help in obtaining venom glands of Daboia from Kolkata. We also thank Prof. Shu, Yu-Yen (Kuangxi Medical University, China) and Prof. R.G. Theakston (Liverpool, UK) for the precious gifts of Daboia venom from Myanmar.

\section{References}

[1] K. Adler, H.M. Smith, S.H. Prince, P. David, D. Chiszar, Russell's Viper: Daboia russelii not Daboia russellii, due to Classical Latin rules, Hamadryad 25 (2000) 83-85.

[2] D.A. Warrell, Geographic and intraspecies variation in the clinical manifestations of envenoming by snakes, in: R.S. Thorpe, W. Wuster, A. Malhotra (Eds.), Venomous Snakes, Clarendon Press, Oxford, U. K., 1997, pp. 189-204.

[3] Than-Tahn, R.A. Hutton, M.L. Khin-Ei-Han, Soe-Soe, Tin-Nu-Swe, R.E. Phillips, D.A. Warrell, Haemostatic disturbances in patients bitten by Russell's viper (Vipera russelli siamensis) in Burma, Br. J. Haematol. 69 (1988) 513-520.

[4] Than-Tahn, N. Francis, Tin-Nu-Swe, Myint-Lwin, Tun-Pe, Soe-Soe, Maung-Maung-Oo, R.E. Phillips, D.A. Warrell, Contribution of focal haemorrhage and microvascular fibrin deposition to fatal envenoming by Russell's viper (Vipera russelli siamensis) in Burma, Acta Trop. 46 (1989) $23-38$.

[5] Tin-Nu-Swe, Tin-Tun, Myint-Lwin, Thein-Than, Tun-Pe, J.I. Robertson, B.J. Leckie, R.E. Phillips, D.A. Warrell, Renal ischemia, transient glomerular leak and acute renal tubular damage in patients envenomed by Russell's vipers (Daboia russelii siamensis) in Myanmar, Trans. R. Soc. Trop. Med. Hyg. 87 (1993) 678-681.

[6] A.V. Kumar, T.V. Gowda, Novel non-enzymatic toxic peptide of Daboia russelii (Eastern region) venom renders commercial polyvalent antivenom ineffective, Toxicon 47 (2006) 398-408.

[7] N.B. Prasad, B. Uma, K.G.S. Bhatt, T.V. Gowda, Comparative characterization of the variable properties of venoms of Russell's viper (Vipera russelli) venom from Indian peninsula, Biochim. Biophys. Acta 1428 (1999) 121-136.

[8] A.K. Mukherje, S.K. Ghosal, C.R. Maity, Some biochemical properties of Russell's viper (Daboia russelli) venom from Eastern India: correlation with clinico-pathological manifestation in Russell's viper bite, Toxicon 38 (2000) 163-175.

[9] A.K. Chakraborty, R.H. Hall, A.C. Ghose, Purification and characterization of a potent hemolytic toxin with phospholipase $\mathrm{A}_{2}$ activity from the venom of Indian Russell's viper, Mol. Cell. Biochem. 237 (2002) 95-102.

[10] J.M. Danse, S. Gasparini, A. Menez, Molecular biology of snake venom phospholipases $A_{2}$, in: R.M. Kini (Ed.), Venom Phospholipase $A_{2}$ Enzyme: Structure, Function and Mechanism, J. Wiley and Sons, London, 1997, pp. 29-71.

[11] Y.H. Chen, Y.M. Wang, M.J. Hseu, I.H. Tsai, Molecular evolution and structure-function relationships of crotoxin-like and asparagine 6containing phospholipases $A_{2}$ in pit viper venoms, Biochem. J. 381 (2004) 25-34.

[12] I.H. Tsai, Y.M. Wang, Y.H. Chen, T.S. Tsai, M.C. Tu, Venom phospholipases $\mathrm{A}_{2}$ of bamboo viper (Trimeresurus stejnegeri): molecular characterization, geographic variations and evidence of multiple ancestries, Biochem. J. 377 (2004) 215-223.

[13] T. Ogawa, M. Kitajima, K.I. Nakashima, Y. Sakaki, M. Ohno, Molecular evolution of group II phospholipase $A_{2}$, J. Mol. Evol. 41 (1995) 867-877.

[14] Y.M. Wang, P.J. Lu, C.L. Ho, I.H. Tsai, Characterization and molecular cloning of neurotoxic phospholipases $\mathrm{A}_{2}$ from Taiwan viper (Vipera russelli formosensis), Eur. J. Biochem. 209 (1992) 635-641.

[15] I. Nuchprayoon, A. Sai-Ngam, S. Suntrarachun, J. Noiphrom, N. Pakmanee, L. Chanhome, S. Nuchprayoon, V. Sitprija, Molecular cloning of phospholipase $\mathrm{A}_{2}$ from a Thai Russell's viper venom gland cDNA library, J. Med. Assos. Thail., Suppl. 84 (Suppl 1) (2001) S99-S105.

[16] V.T. Gowda, J. Schmidt, J.L. Middlebrook, Primary sequence determination of the most basic myonecrotic phospholipase $\mathrm{A}_{2}$ from the venom of Vipera russelli, Toxicon 32 (1994) 665-673.

[17] Maung-Maung-Thwin, P. Gopalakrishnakone, R. Yuen, C.H. Tan, A major lethal factor of the venom of Burmese Russell's viper (Daboia russelii siamensis): isolation, $\mathrm{N}$-terminal sequencing and biological activities of daboiatoxin, Toxicon 33 (1995) 63-76.

[18] Maung-Maung-Thwin, P. Gopalakrishnakone, R. Yuen, C.H. Tan, Synaptosomal binding of ${ }^{125}$ I-labelled daboiatoxin, a new phospholipase $\mathrm{A}_{2}$ neurotoxin from the venom of Daboia russelli siamensis, Toxicon 34 (1996) 183-199.

[19] I.H. Tsai, P.J. Lu, Y.C. Su, Two types of Russell's viper revealed by variation in phospholipases $\mathrm{A}_{2}$ from venom of the sub-species, Toxicon 34 (1996) 99-109.

[20] M.M. Bradford, A rapid and sensitive method for the quantitation of microgram quantities of protein utilizing the principle of protein-dye binding, Anal. Biochem. 72 (1976) 248-254.

[21] M.W. Hunkapiller, L.E. Hood, Analysis of phenylthiohydantoins by ultrasensitive gradient high-performance liquid chromatography, Methods Enzymol. 91 (1983) 486-493.

[22] K.B. Mullis, F.A. Faloona, Specific synthesis of DNA in vitro via a polymerase-catalyzed chain reaction, Methods Enzymol. 155 (1987) 335-350.

[23] T. Maniatis, E.F. Fritsch, J. Sambrook, Molecular Cloning, a Laboratory Manual, 2nd ed.Cold Spring Harbor Laboratory, Cold Spring Harbor, NY, 1989.

[24] G.P. Jayanthi, T.V. Gowda, Synergistic interaction of a protease and protease inhibitors from Russell's viper (Vipera russelli) venom, Toxicon 28 (1990) 65-74.

[25] S.F. Altschul, T.L. Madden, A.A. Schaffer, J. Zhang, Z. Zhang, W. Miller, D.J. Lipman, Gapped BLAST and PSI-BLAST: a new generation of protein database search programs, Nucleic Acids Res. 25 (1997) 3389-3402.

[26] J. Felsenstein, PHYLIP: the PHYLogeny Inference Package, version 3.573. Computer Program Distributed by the U. of Washington, Dept. of Genetics, Seattle, U.S.A., 1992.

[27] J. Felsenstein, Confidence limits on phylogenies: an approach using the bootstrap, Evolution 39 (1985) 783-791.

[28] F. Radvanyi, B. Saliou, C. Bon, P.N. Strong, The interaction between the presynaptic phospholipase neurotoxins beta-bungarotoxin and crotoxin and mixed detergent- phosphatidylcholine micelles. A comparison with non-neurotoxic snake venom phospholipases $\mathrm{A}_{2}$, J. Biol. Chem. 262 (1987) 8966-8974.

[29] T. Petan, I. Krizaj, M.H. Gelb, J. Pungercar, Ammodytoxins, potent presynaptic neurotoxins, are also highly efficient phospholipase $\mathrm{A}_{2}$ enzymes, Biochemistry 44 (2005) 12535-12545.

[30] E. Carredano, B. Westerlund, B. Persson, M. Saarinen, S. Ramaswamy, D. Eaker, H. Eklund, The three-dimensional structures of two toxins from snake venom throw light on the anticoagulant and neurotoxic sites of phospholipase $A_{2}$, Toxicon 36 (1998) 75-92.

[31] H.C. Huang, C.Y. Lee, Isolation and pharmacological properties of phospholipases $\mathrm{A}_{2}$ from Vipem russelli (Russell's viper) snake venom, Toxicon 22 (1984) 207-217.

[32] W. Wuster, S. Otsuka, R.S. Thorpe, A. Malhotra, Morphological variation in Russell's viper in Burma and Thailand, Herpetol. J. 2 (1992) 99-101.

[33] Win-Aung, N. Pakmanee, O. Khow, C. Nambut, V. Sitprija, Cross neutralization of the lethal activities of Myanmar and Thai Russell's 
viper venoms by Thai and Myanmar antivenoms, J. Nat. Toxins 10 (2001) $335-342$.

[34] F. Bossuyt, M.C. Milinkovitch, Amphibians as indicators of early Tertiary "Out-of-India" dispersal of vertebrate, Science 292 (2001) 93-95.

[35] P. Lenk, S. Kalyabina, M. Wink, U. Joger, Evolutionary relationships among the true vipers (Reptilia: Viperidae) inferred from mitochondrial DNA sequences, Mol. Phylogenet. Evol. 19 (2001) 94-104.

[36] T. Garrigues, C. Dauga, E. Ferquel, V. Choumet, A.B. Failloux, Molecular phylogeny of Vipera Laurenti, 1768 and the related genera Macrovipera and Daboia (Gray, 1842), with comments about neurotoxic Vipera aspis aspis populations, Mol. Phylogenet. Evol. 35 (2005) 35-47.

[37] E. Siigur, A. Aaspollu, K. Trummal, K. Tonismagi, I. Tammiste, N. Kalkkinen, J. Siigur, Factor X activator from Vipera lebetina venom is synthesized from different genes, Biochim. Biophys. Acta 1702 (2004) 41-51.

[38] E. Siigur, A. Aaspollu, J. Siigur, Molecular cloning and sequence analysis of a cDNA for factor $\mathrm{V}$ activating enzyme, Biochem. Biophys. Res. Commun. 262 (1999) 328-332.

[39] R.W.P. Master, F. Kornalik, Biochemical differences in yellow and white venoms of Vipera ammodytes and Russell's viper, J. Biol. Chem. 240 (1965) $139-142$.

[40] V. Jan, R.C. Maroun, A. Robbe-Vincent, L. De Haro, V. Choumet, Toxicity evolution of Vipera aspis aspis venom: identification and molecular modeling of a novel phospholipase $\mathrm{A}_{2}$ heterodimer neurotoxin, FEBS Lett. 527 (2002) 263-268.

[41] I. Guillemin, C. Bouchier, T. Garrigues, A. Wisner, V. Choumet, Sequences and structural organization of phospholipase $\mathrm{A}_{2}$ genes from Vipera aspis aspis, V. aspis zinnikeri and Vipera berus berus venom. Identification of the origin of a new viper population based on ammodytin I1 heterogeneity, Eur. J. Biochem. 270 (2003) 2697-2706.

[42] D. Kordis, A. Bdolah, F. Gubensek, Positive Darwinian selection in Vipera palaestinae phospholipase $\mathrm{A}_{2}$ genes is unexpectedly limited to the third exon, Biochem. Biophys. Res. Commun. 251 (1998) 613-619.
[43] R.M. Kini, Y.M. Chan, Accelerated evolution and molecular surface of venom phospholipase A2 enzymes, J. Mol. Evol. 48 (1999) 125-132.

[44] W. Wuster, S. Otsuka, A. Malhotra, R.S. Thorpe, Population systematics of Russell's viper: a multivariate study, Biol. J. Linn. Soc. 47 (1992) 97-113.

[45] J.C. Daltry, W. Wuster, R.S. Thorpe, The role of ecology in determining venom variation in Malayan pit viper, C. rhodostoma, in: R.S. Thorpe, W. Wuster, A. Malhotra (Eds.), Venomous Snakes, Clarendon Press, Oxford, UK, 1997, pp. 155-172.

[46] D.L. Scott, S.P. White, Z. Otwinowski, W. Yuan, M.H. Gelb, P.B. Sigler, Interfacial catalysis: the mechanism of phospholipase $A_{2}$, Science 250 (1990) 1541-1546.

[47] X. Liu, H. Zhu, B. Huang, J. Rogers, B.Z. Yu, A. Kumar, M.K. Jain, M. Sundaralingam, M.D. Tsai, Phospholipase $\mathrm{A}_{2}$ engineering. Probing the structural and functional roles of $\mathrm{N}$-terminal residues with site-directed mutagenesis, X-ray, and NMR, Biochemistry 34 (1995) 7322-7334.

[48] M. Rigoni, P. Caccin, S. Gschmeissner, G. Koster, A.D. Postle, S.G. Rossetto, C. Montecucco, Neuroscience: Equivalent effects of snake phospholipase $\mathrm{A}_{2}$ neurotoxins and lysophospholipid-fatty acid mixtures, Science 310 (5754) (2005) 1678-1680.

[49] M. Rouault, L.D. Rash, P. Escoubas, E. Boilard, J. Bollinger, B. Lomonte, T. Maurin, C. Guillaume, S. Canaan, C. Deregnaucourt, J. Schrevel, A. Doglio, J.M. Gutierrez, M. Lazdunski, M.H. Gelb, G. Lambeau, Neurotoxicity and other pharmacological activities of the snake venom phospholipase $\mathrm{A}_{2}$ OS2: the N-terminal region is more important than enzymatic activity, Biochemistry 45 (2006) 5800-5816.

[50] I.H. Tsai, Y.M. Wang, Effect of site directed mutagenesis on the activity of recombinant trimucrotoxin, a neurotoxic phospholipase from Trimeresurus mucrosquamatus venom, Toxicon 36 (1998) 1591-1598.

[51] R. Renetseder, S. Brunie, B.W. Dijkstra, J. Drenth, P.B. Sigler, A comparison of the crystal structures of phospholipase $\mathrm{A}_{2}$ from bovine pancreas and Crotalus atrox venom, J. Biol. Chem. 260 (1985) 11627-11636. 